\section{Control of plant height by 24 alleles at 12 quantitative trait loci in rice}

\author{
Yuxiang Zeng ${ }^{1}$, Yuan Chen ${ }^{1}$, Zhijuan Ji ${ }^{1}$, Yan Liang ${ }^{1}$, Anfu Zheng ${ }^{1}$, \\ Zhihua Wen ${ }^{1}$ and Changdeng Yang ${ }^{{ }^{*}}$
}

\begin{abstract}
Plant height (PH) is controlled by quantitative trait loci (QTLS) in rice. In the present study, a recombinant inbred line population developed by crossing two rice cultivars, Lemont and Yangdao4, was grown in eight environments for QTL analysis. Multiple interval mapping detected 53 PH-QTLs, 39 of which clustered at 12 chromosome regions/putative loci. An examination of the 12 putative loci identified 24 alleles that are simultaneously involved in controlling $\mathrm{PH}$. Linear regression analyses suggested that these 24 alleles function additively across the 12 loci to control PH, and plants carrying more PH-increasing alleles at the 12 loci were taller than those carrying more PH-decreasing alleles. Multiple comparison tests indicated that the effect of a single allele at the 12 loci was small and that multiple alleles must be pyramided to attain a statistically significant effect. The closest markers to the 12 loci can be used directly in marker-assisted breeding to manipulate $\mathrm{PH}$.
\end{abstract}

Keywords: rice, plant height, QTL, allele, recombinant inbred line

\section{INTRODUCTION}

In rice (Oryza sativa L.) production, the occurrence of lodging is common during the grain fill period after strong winds accompanied by heavy rain (Sowadan et al. 2018). Lodging reduces the yield, quality of produce, and mechanical harvesting efficiency (Weber and Fehr 1966, Sowadan et al. 2018). Plant height (PH), an important trait affecting lodging, has been the primary target for improving resistance against lodging (Sowadan et al. 2018). During the 1960s, rice varieties with reduced PH (semidwarf varieties) and improved lodging resistance were developed that strongly increased the rice yield and initiated the rice green revolution in Asia (Khush 1999).

Rice PH is controlled by quantitative trait loci (QTLs) (Huang et al. 1996). Thousands of QTLs for PH have been mapped on all 12 rice chromosomes using QTL analysis (Li et al. 1995, Huang et al. 1996, Feng et al. 2011, Wen et al. 2015, Han et al. 2017) or association mapping (Zhou et al. 2016, Sowadan et al. 2018). Genes controlling rice PH have been characterized or cloned, including 17 genes involved in the gibberellin pathways, 25 genes involved in the brassinosteroid pathways, 10 genes involved in the strigolactone pathways, and 22 genes involved in other phytohormone pathways (Liu et al. 2018). The famous green revolution gene $s d 1$ was cloned and characterized by three independent groups in 2002 (Monna et al. 2002, Sasaki et al. 2002, Spielmeyer et al. 2002).
Crop Breeding and Applied Biotechnology 19: 200-207, 2019 Brazilian Society of Plant Breeding. Printed in Brazil http://dx.doi.org/10.1590/198470332019v19n2a28

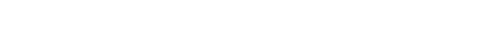


Recombinant inbred line (RIL) populations are often used to detect and confirm PH-QTLs. Although QTLs related to PH have been identified using different RIL populations, it is still largely unknown how the different QTLS detected within a specific population control PH and whether these QTLs can be used simultaneously to manipulate $\mathrm{PH}$. In this study, we used a RIL population developed by crossing two rice cultivars, Lemont and Yangdao4. The objectives were to use the Lemont ' Yangdao4 RIL population to (1) detect QTLs related to PH, (2) examine how the combination of different alleles in different QTLs determines PH, and (3) explore molecular markers that can be used to manipulate $\mathrm{PH}$.

\section{MATERIAL AND METHODS}

\section{RIL population}

The present study used a rice RIL population consisting of 219 lines developed via single seed descent by crossing Lemont, an American japonica variety, with Yangdao4, a Chinese indica variety. The Lemont ' Yangdao4 RIL population was initially developed for mapping sheath blight resistance QTLS.

The RIL population was sown in eight different environments on the farm of the China National Rice Research Institute (CNRRI) in Fuyang (lat $119^{\circ} 95^{\prime} \mathrm{E}$, long $30^{\circ} 07^{\prime} \mathrm{N}$ ), Hangzhou, or the CNRRI trial station in Lingshui (lat $110^{\circ} 02^{\prime} \mathrm{E}$, long $18^{\circ}$ 48' N), Hainan, for QTL analysis: (1) May 24, 2013, in Hangzhou; (2) November 23, 2016, in Hainan; (3) May 29, 2017, in Hangzhou; (4) June 7, 2017, in Hangzhou; (5) June 30, 2017, in Hangzhou; (6) November 25, 2017, in Hainan; (7) May 23, 2018, in Hangzhou; and (8) June 2, 2018, in Hangzhou.

\section{Measurement of PH}

The 219 lines of the RIL population were planted as 219 plots in eight different environments as mentioned above for measurement of PH. Plot locations were randomized. Eighteen individual plants of each line were planted in every environment. The 18 plants were arranged in three rows of six plants each, separated by $20 \mathrm{~cm}$ between rows and 17 $\mathrm{cm}$ between the plants of each row. Five plants of each line were randomly selected for measurement of $\mathrm{PH}$, which was measured from the soil surface to the tip of the tallest panicle (not including awn). The average PH of five plants of each line was used for QTL analysis.

\section{Construction of genetic linkage map}

A total of 208 polymorphic markers covering the 12 rice chromosomes were used to construct a genetic linkage map using Mapmaker/EXP version 3.0 (Lander et al. 1987). The 208 polymorphic markers consisted of insertiondeletion markers with ' $D$ ' or ' $G$ ' prefix and simple sequence repeat markers with 'RM' prefix. The names of the 208 markers are provided in Figure 1 and Figure 2. The primer sequences of the insertion-deletion ' $D$ ' markers have been reported by Zeng et al. (2013). The sequences of the simple sequence repeat 'RM' markers can be found at the Gramene website (www.gramene.org). The sequences of the insertion-deletion ' $G$ ' markers are as follows: GW1C (5'-AGCGTTCGCAACTTCG-3', 5'-ATCCGTTCCGCTTCCA-3'), GL31C (5'-ATGGTCGGAGTTGTGGAAGTG3', 5' -CATCGGTGCATCGTGGG-3'), GL31F (5'-CTGCACGGAGCGCATAGA-3', 5' -TTGGAGTGGTTGGGAGACG-3'), GW32F (5'-TGACTCCACCAGAACC-3', 5'-AAACCCAAACACGAAT-3'), GL32G (5' -CTGCGATTGTATCTCACTT-3', 5'-CTCATGGAGGACAGAAGA-3'), GL4F (5'-GAACAGCGTGTATTGGT-3', 5'-GAGGGAAGAAGAGGAAA-3'), and GL4J (5'-TACCGTTCGAGTAAACCC-3', 5'-CTGCTTCCCTTGTGCTT-3').

A genetic linkage map was constructed using 208 polymorphic markers to assay leaves of the 219 lines of the Lemont ' Yangdao4 RIL population. DNA extraction and PCR protocol followed Ye et al. (2017).

\section{Multiple interval mapping and statistical analysis}

Multiple interval mapping (MIM) was run in Windows QTL Cartographer 2.5 software (Wang et al. 2012). The MIM model used for QTL analysis followed Zeng et al. (2016). Detection of two or more QTLs in the same marker interval was defined as a QTL cluster. The statistical analysis, including linear regression analysis and Duncan's multiple range test, was performed using SAS 8.01 software (SAS Institute, Cary, NC, USA). 


\section{RESULTS AND DISCUSSION}

\section{QTLs identified using the MIM method}

A genetic linkage map was constructed with 208 polymorphic markers covering 12 rice chromosomes. The linkage map represented a total of $2228.0 \mathrm{cM}$ with an average of $11.4 \mathrm{cM}$ between adjacent markers (Figures 1 and 2).

QTL analysis detected a total of $53 \mathrm{PH}-\mathrm{QTLs}$ in eight environments using MIM (Figures 1 and 2). These QTLs are mapped on 10 of the 12 chromosomes. Forty-eight of the 53 QTLs accounted for less than $10 \%$ of the phenotypic variation individually, indicating that most of the QTLs had a minor effect. Five of the 53 QTLs had a relatively large effect that accounted individually for 12.9-15.2\% of the phenotypic variation: $q P H 10.2$ (detected in 2016 in Hainan), $q P H 2.2$ (detected on May 29, 2017, in Hangzhou), qPH12.3 (detected on June 7, 2017, in Hangzhou), qPH12.1 (detected on June 30, 2017, in Hangzhou), and $9 P H 12.1$ (detected on June 2, 2018, in Hangzhou) accounted for 13.0\%, 12.9\%, 15.2\%, $13.0 \%$, and $12.9 \%$ of the phenotypic variations, respectively.

It was found that most of the QTLs were clustered in some QTL-rich regions: thirty-nine QTLs were clustered in twelve QTL-rich regions (Table 1). We focused on the 12 QTL clusters/putative loci because a QTL cluster consists of at least two QTLs and is expected to be more stable than a QTL detected only once.
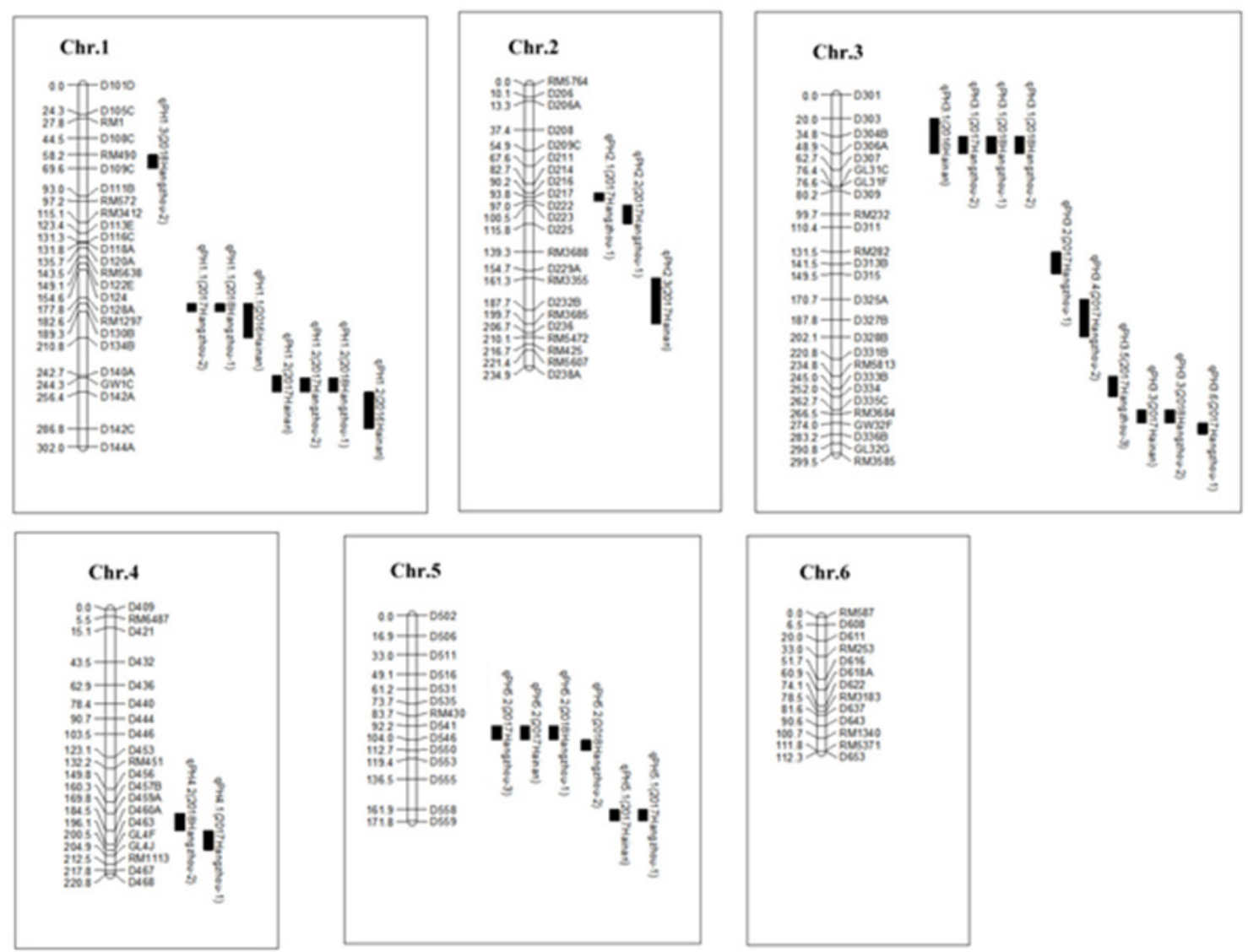

Figure 1. Genetic linkage map (chromosomes 1-6) constructed using the Lemont'Yangdao4 recombinant inbred line population consisting of 219 lines and QTLs responsible for plant height detected using multiple interval mapping in eight environments. The environments are listed in parentheses after the QTL name at the right of the chromosome bars. 2017 Hangzhou-1: sown in May 29, 2017, in Hangzhou; 2017 Hangzhou-2: sown in June 7, 2017, in Hangzhou; 2017 Hangzhou-3: sown in June 30, 2017, in Hangzhou; 2018 Hangzhou-1: sown in May 23, 2018, in Hangzhou; and 2018 Hangzhou-2: sown in June 2, 2018, in Hangzhou. 


\section{Control of PH by 24 alleles at 12 putative loci}

We examined all 12 putative loci carefully. At five putative loci (qPH1.2, qPH3.3, qPH8.2, qPH9.1, and $q P H 10.2)$, the alleles for increased height were from Lemont; at the other seven loci ( $q P H 1.1, q P H 3.1, q P H 5.2, q P H 5.1, q P H 11.1, q P H 12.1$, and $q P H 12.3$ ), the alleles for increased height were from Yangdao4 (Table 1). The $12 \mathrm{PH}$-increasing alleles were $q P H 1.2 L E$,
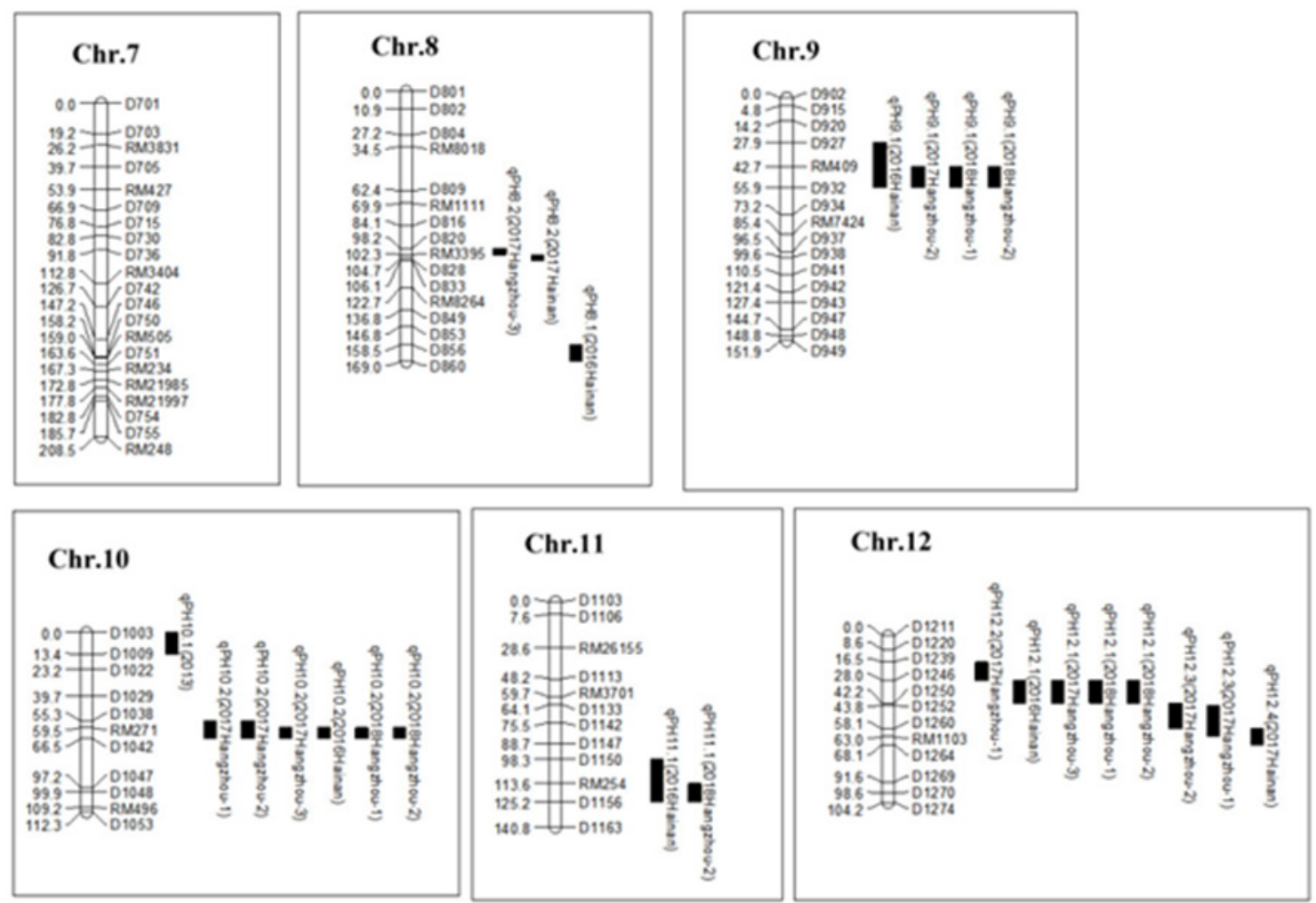

Figure 2. Genetic linkage map (chromosomes 7-12) constructed using the Lemont'Yangdao4 recombinant inbred line mapping population consisting of 219 lines and QTLs responsible for plant height detected using multiple interval mapping in eight environments. The environments are listed in parentheses after the QTL name at the right of the chromosome bars. 2017 Hangzhou-1: sown in May 29, 2017, in Hangzhou; 2017 Hangzhou-2: sown in June 7, 2017, in Hangzhou; 2017 Hangzhou-3: sown in June 30, 2017, in Hangzhou; 2018 Hangzhou-1: sown in May 23, 2018, in Hangzhou; and 2018 Hangzhou-2: sown in June 2, 2018, in Hangzhou.

Table 1. Thirty-nine plant height QTLs identified by multiple interval mapping were clustered in twelve QTL-rich regions/putative loci

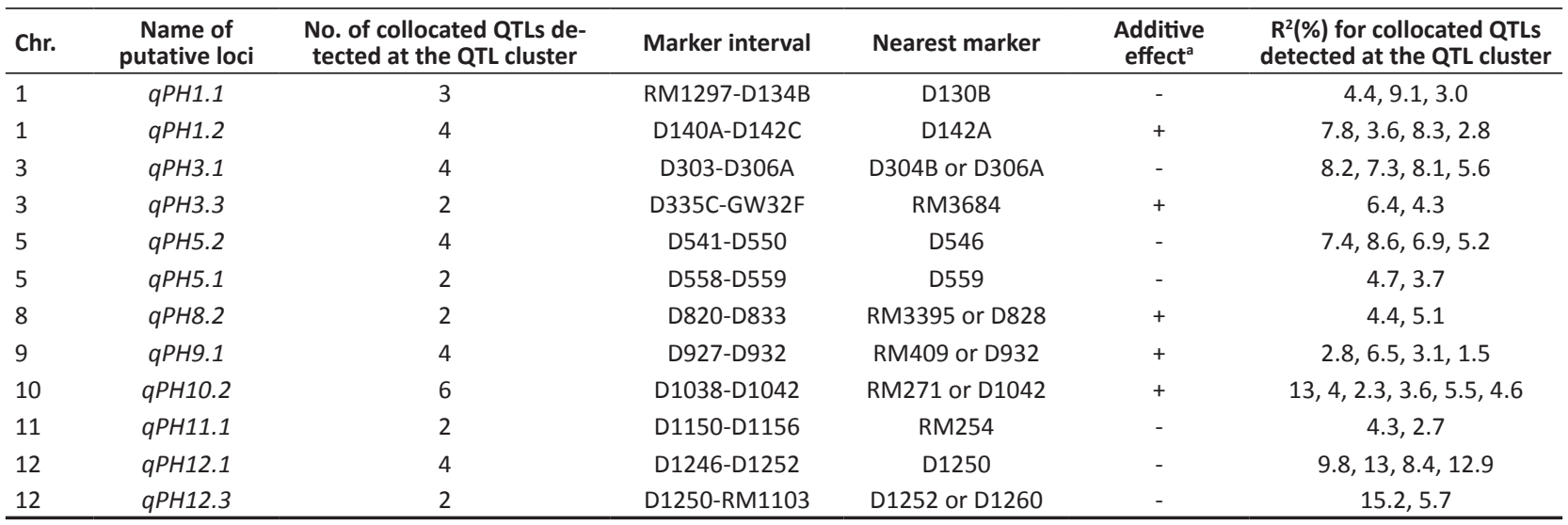

${ }^{\mathrm{a}}$ A negative additive effect (-) indicated that the allele from Yangdao4 increased plant height, whereas a positive additive effect (+) indicated that the allele from Lemont increased plant height. 
qPH3.3LE, qPH8.2LE, qPH9.1LE, qPH10.2LE, qPH1.1YD, qPH3.1YD, qPH5.2YD, qPH5.1YD, qPH11.1YD, qPH12.1YD, and $q P H 12.3 Y D$, and the $12 \mathrm{PH}$-decreasing alleles were $q P H 1.2 Y D, q P H 3.3 Y D, q P H 8.2 Y D, q P H 9.1 Y D, q P H 10.2 Y D, q P H 1.1 L E$, qPH3.1LE, qPH5.2LE, qPH5.1LE, qPH11.1LE, qPH12.1LE, and $q P H 12.3 L E$; 'LE' or 'YD' suffixes in QTL names indicate if it was inherited from 'Lemont' or 'Yangdao4', respectively.

First, we calculated how many $\mathrm{PH}$-increasing alleles and $\mathrm{PH}$-decreasing alleles existed at the 12 putative loci for different lines of the Lemont ' Yangdao4 RIL population. The nearest markers to each of the 12 loci were selected to represent the 12 loci (Table 1). The 12 markers are D130B, D142A, D306A, RM3684, D546, D559, RM3395, RM409, RM271, RM254, D1250, and D1260. Second, we used regression analysis to test in the RIL population whether more $\mathrm{PH}$-increasing alleles in a line would lead to a taller $\mathrm{PH}$ phenotype. Regression analysis using data in eight environments proved this assumption and yielded eight equations (May 2013, Hangzhou: $\mathrm{F}=13.2, P=0.0004$; November 2016, Hainan: $\mathrm{F}=$ 92.0, $P<$ 0.0001; May 29, 2017, Hangzhou: $\mathrm{F}=74.2, P<0.0001$; June 7, 2017, Hangzhou: $\mathrm{F}=86.5, P<0.0001$; June 30, 2017, Hangzhou: $F=123.6, P<0.0001$; November 2017, Hainan: $F=76.0, P<0.0001$; May 23, 2018, Hangzhou: $F=$ 105.9, $P<0.0001$; and June 2, 2018, Hangzhou: $F=96.6, P<0.0001$ ). The eight equations are provided in Figure 3 and Figure 4. These results indicated that (1) plants carrying more height-increasing alleles at the 12 loci were taller than those carrying more height-decreasing alleles and (2) PH was controlled at least by the 12 loci simultaneously.

\section{Effect of a single allele at the 12 putative loci}

There are 24 alleles at the 12 putative loci. We examined the extent of the effect of a single allele on the PH phenotype by using the following method. First, we classified the different lines of the RIL population into 8 different groups based on the number of height-increasing alleles each carried at the 12 putative loci (Figures 5 and 6 ). However, because of the limited number of lines within the RIL population, lines with more than 20 height-increasing alleles or less than 6 cannot be found. Second, we used Duncan's multiple range test $(P=0.05)$ to compare the differences among the 8 different groups (Figures 5 and 6 ). The analysis showed that lines carrying 6, 8, or 10 height-increasing alleles did not significantly differ in PH among the 8 environments. Plants carrying 12 or 14 height-increasing alleles did not have a significantly
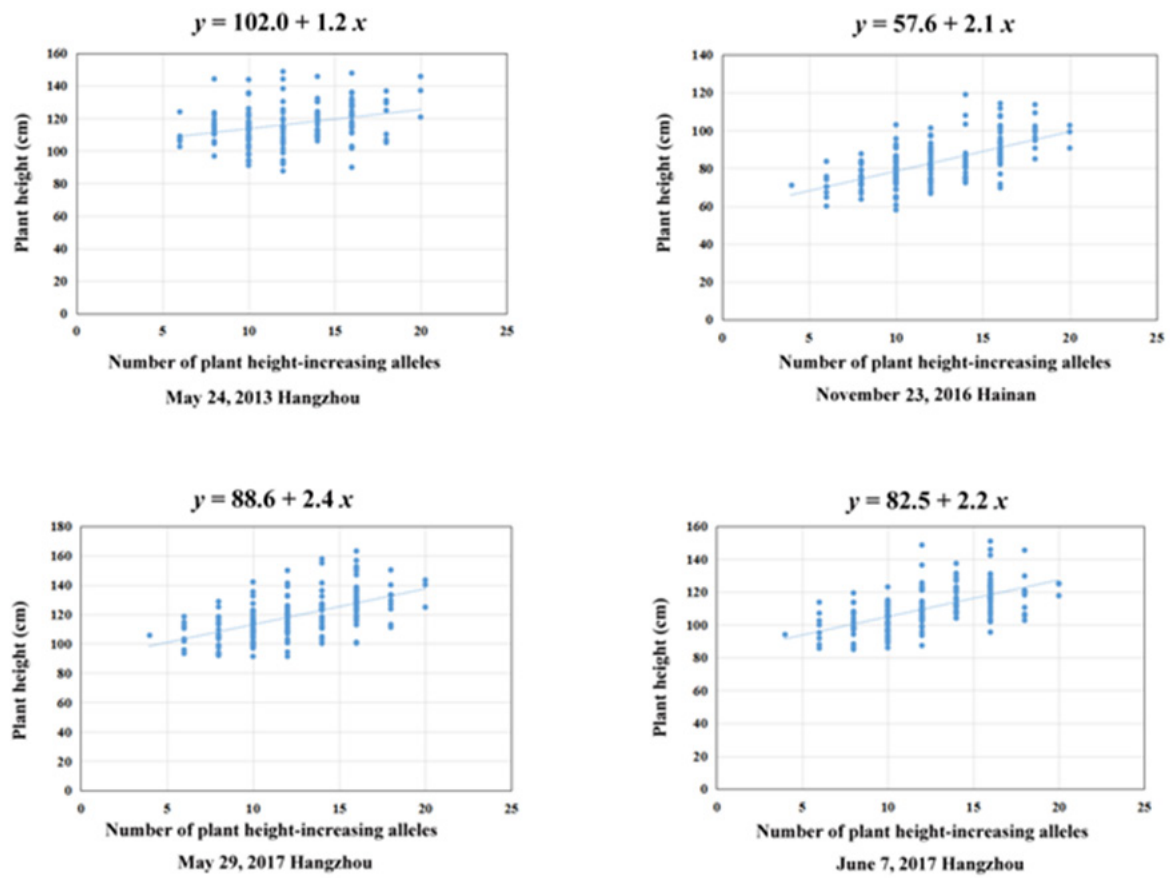

Figure 3. Regression analysis between plant height and number of plant height-increasing alleles at 12 loci ( $q P H 1.1, q P H 1.2, q P H 3.1$, qPH3.3, qPH5.2, qPH5.1, qPH8.2, qPH9.1, qPH10.2, PH11.1, qPH12.1, and qPH12.3) using 219 lines of the Lemont ' Yangdao4 RIL population planted in four environments: (1) sown on May 24, 2013, in Hangzhou; (2) sown on November 23, 2016, in Hainan; (3) sown on May 29, 2017, in Hangzhou; and (4) sown on June 7, 2017, in Hangzhou. 

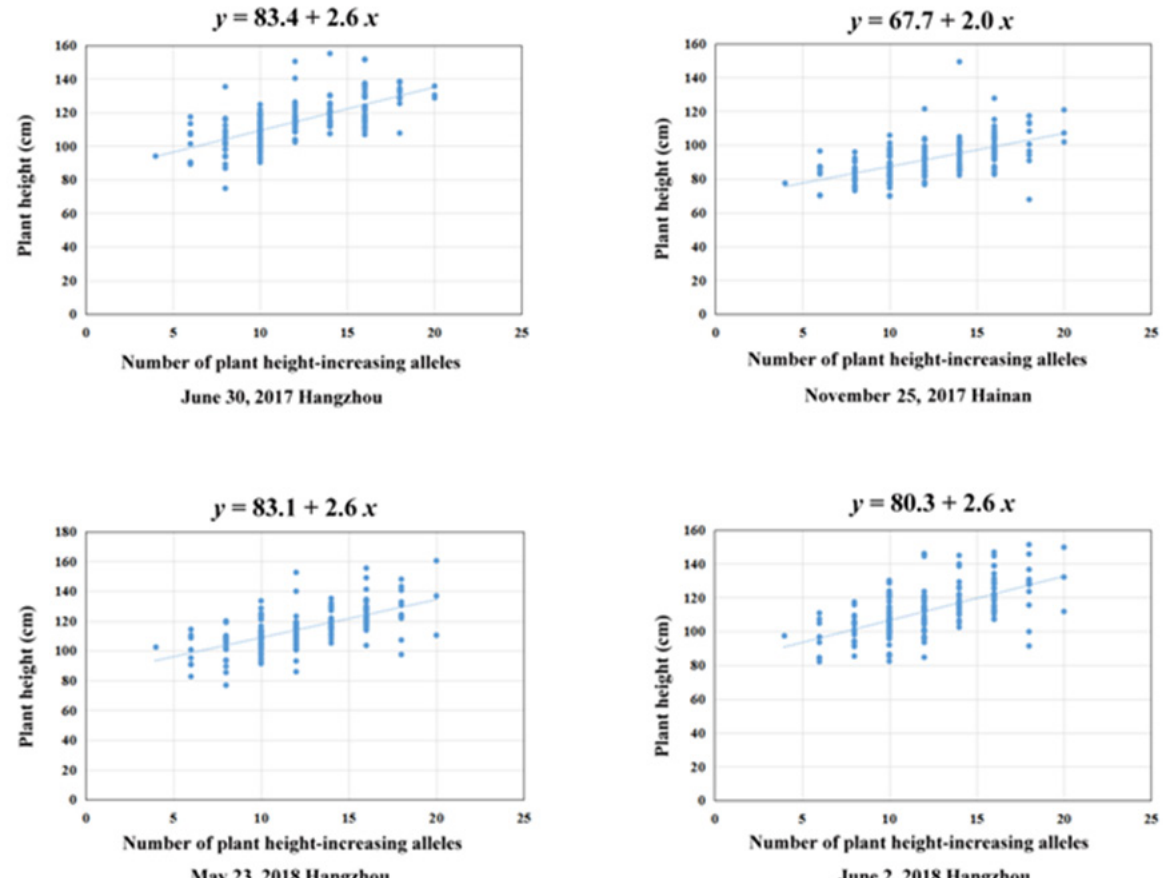

Figure 4. Regression analysis between plant height and number of plant height-increasing alleles at 12 loci $(q P H 1.1, q P H 1.2, q P H 3.1$, qPH3.3, qPH5.2, qPH5.1, qPH8.2, qPH9.1, qPH10.2, PH11.1, qPH12.1, and qPH12.3) using 219 lines of the Lemont 'Yangdao4 RIL population planted in four environments: (1) sown in June 30, 2017, in Hangzhou; (2) sown in November 25, 2017, in Hainan; (3) sown in May 23, 2018, in Hangzhou; and (4) sown in June 2, 2018, in Hangzhou.
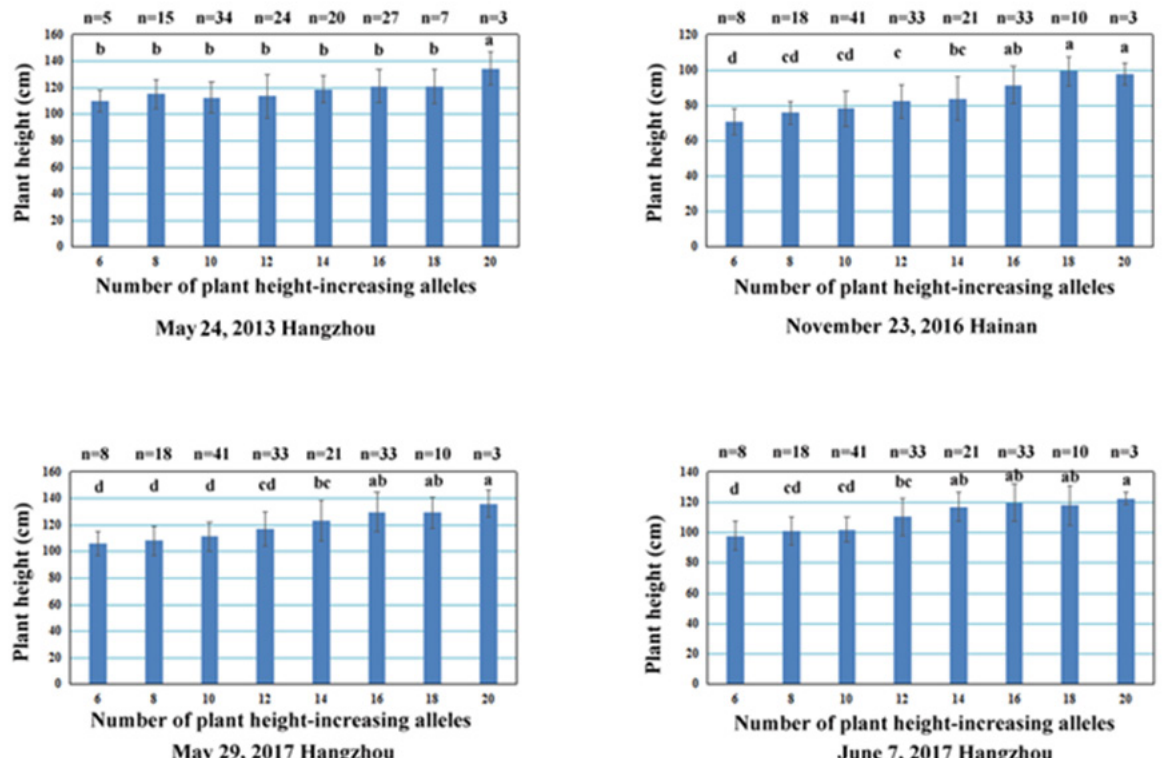

Figure 5. Multiple comparison test (Duncan's, $P=0.05)$ among different lines carrying different numbers of plant height-increasing alleles at the Lemont'Yangdao4 recombinant inbred line population planted in four different environments: (1) sown on May 24, 2013, in Hangzhou; (2) sown on November 23, 2016, in Hainan; (3) sown on May 29, 2017, in Hangzhou; and (4) sown on June 7, 2017, in Hangzhou. Different lines of the Lemont'Yangdao4 RIL population were classified into 8 different groups based on the number of height-increasing alleles that they carried at 12 loci $(q P H 1.1, q P H 1.2, q P H 3.1, q P H 3.3, q P H 5.2, q P H 5.1, q P H 8.2, q P H 9.1, q P H 10.2$, PH11.1, qPH12.1, and $q P H 12.3)$. 

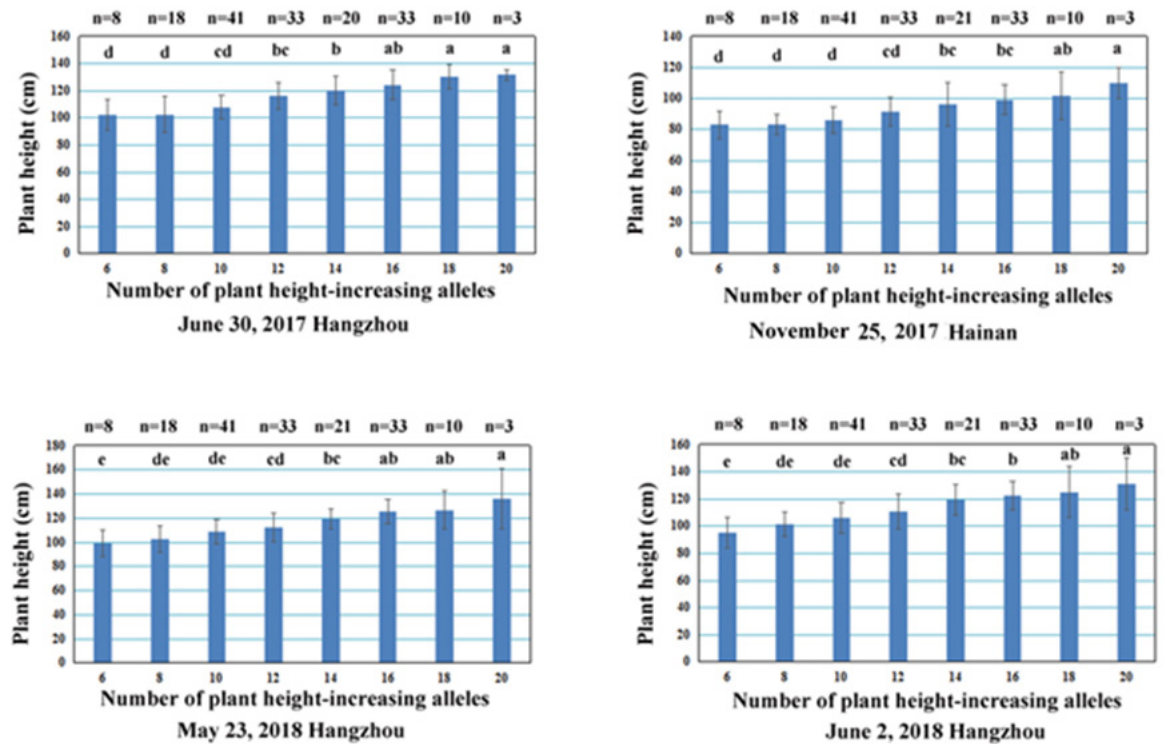

Figure 6. Multiple comparison test (Duncan's, $P=0.05$ ) among different lines carrying different numbers of plant height-increasing alleles at the Lemont'Yangdao4 recombinant inbred line population planted in four different environments: (1) sown in June 30, 2017, in Hangzhou; (2) sown in November 25, 2017, in Hainan; (3) sown in May 23, 2018, in Hangzhou; and (4) sown in June 2, 2018, in Hangzhou. Different lines of the Lemont'Yangdao4 RIL population were classified into 8 different groups based on the number of height-increasing alleles that they carried at 12 loci (qPH1.1, qPH1.2, qPH3.1, qPH3.3, qPH5.2, qPH5.1, qPH8.2, qPH9.1, qPH10.2, $P H 11.1, q P H 12.1$, and $q P H 12.3)$.

different PH. Similarly, plants carrying 14 or 16 height-increasing alleles did not have a significantly different PH (Figure 5 and 6). These observations indicated that the contribution of each of the 24 alleles at the 12 loci was incremental, one or several alleles at the 12 loci cannot achieve a significant contribution to the final PH phenotype. This finding was consistent with the MIM mapping results that most of the identified QTLs had only a minor effect. Therefore, if the 12 loci would be used in marker-assisted selection, we suggest that all 12 loci should be included to have a significant effect.

\section{Complex regulatory network for rice $\mathrm{PH}$}

Previous reports indicated that genes can be pyramided to affect PH. Tomita (2012) showed that pyramiding two semidwarfing genes, $d 60$ and $s d 1$, resulted in a PH that was shorter than that using either $d 60$ or $s d 1$. Hu et al. (2013) reported that a double mutant of tud1/d61 was shorter than either tud1 or $d 61$, although tud1 and $d 61$ were already two rice dwarf mutants. Importantly, our present study demonstrated that as much as 12 QTLs can be pyramided to control $\mathrm{PH}$, indicating a complex regulatory network.

Genes controlling PH in rice are involved in the complex regulatory networks of phytohormones, including gibberellins, brassinosteroids, strigolactones, indole-3-acetic acid, abscisic acid, and ethylene (Liu et al. 2018). Other PH regulation pathways with nonhormone factors include cell wall development, cytosolic glutamine synthetic pathway, RNA editing, cell division, ubiquitin-proteasome pathway, and fatty acid metabolism (Liu et al. 2018). It is speculated that the 12 putative loci identified in the present study are involved in multiple regulatory pathways. The markers closest to the 12 loci identified in this study can be used directly in marker-assisted breeding to manipulate PH.

\section{ACKNOWLEDGMENTS}

This work was supported by the National Key R\&D Program (2016YFD0102102), the Zhejiang Provincial Natural Science Foundation (LY16C060002, LQ17C130005), and a fund from the Science Technology Department of Zhejiang Province to the Zhejiang Agricultural Key Breeding Project (2016C02050-4). 


\section{REFERENCES}

Feng Y, Zhai RR, Cao LY, Lin ZC, Wei XH and Cheng SH (2011) QTLs for plant height and heading date in rice under two nitrogen levels. Acta Agronomica Sinica 37: 1525-1532.

Han Z, Hu W, Tan C and Xing Y (2017) QTLs for heading data and plant height under multiple environments in rice. Genetica 145: 67-77.

Hu X, Qian Q, Xu T, Zhang Y, Dong G, Gao T, Xie Q and Xue Y (2013) The U-box E3 ubiquitin ligase TUD1 functions with a heterotrimeric $\mathrm{G} \alpha$ subunit to regulate brassinosteroid-mediated growth in rice. PLoS Genetics 9: e1003391.

Huang N, Courtois B, Khush GS, Lin HX, Wang GL, Wu P and Zheng $\mathrm{K}$ (1996) Association of quantitative trait loci for plant height with major dwarfing genes in rice. Heredity 77: 130-137.

Khush GS (1999) Green revolution: preparing for the $21^{\text {st }}$ century. Genome 42: 646-655.

Lander ES, Green P, Abrahamson J, Barlow A, Daly MJ, Lincoln SE and Etoh T (1987) MAPMAKER: an interactive computer package for constructing primary genetic linkage maps of experimental and natural populations. Genomics 1:174-181.

Li ZK, Pinson SRM, Stansel JW and Park WD (1995) Identification of quantitative trait loci (QTLs) for heading date and plant height in cultivated rice (Oryzasative L.). Theoretical and Applied Genetics 91: 374-381.

Liu F, Wang P, Zhang X, Li X, Yan X, Fu D and Wu G (2018) The genetic and molecular basis of crop height based on a rice model. Planta 247: 1-26.

Monna L, Kitazawa N, Yoshino R, Suzuki J, Masuda H, Maehara Y, Tanji M, Sato M, Nasu S and Minobe $Y$ (2002) Positional cloning of rice semidwarfing gene, $s d-1$ : rice 'green revolution gene' encodes a mutant enzyme involved in gibberellin synthesis. DNA Research 9: 11-17.

Sasaki A, Ashikari M, Ueguchi-Tanaka M, Itoh H, Nishimura A, Swapan D, Ishiyama K, Saito T, Kobayashi M and Khush G (2002) Green revolution: a mutant gibberellin-synthesis gene in rice. Nature $\mathbf{4 1 6}$ : 701-702.
Sowadan O, Li D, Zhang Y, Zhu S, Hu X, Bhanbhro LB, Edzesi WM, Dang X and Hong D (2018) Mining of favorable alleles for lodging resistance traits in rice (Oryza sativa) through association mapping. Planta 248: 155-169.

Spielmeyer W, Ellis MH and Chandler PM (2002) Semidwarf (sd-1), "green revolution" rice, contains a defective gibberellin 20-oxidase gene. Proceedings of the National Academy of Sciences of the United States of America 99: 9043-9048.

Tomita M (2012) Combining two semidwarfing genes $d 60$ and $s d 1$ for reduced height in 'Minihikari', a new rice germplasm in the 'Koshihikari' genetic background. Genetic Research Cambridge 94: 235-244.

Wang SC, Basten J and Zeng ZB (2012) Windows QTL Cartographer 2.5. Department of Statistics, North Carolina State University, Raleigh, NC. Available at <http://statgen.ncsu.edu/qtlcart/WQTLCart.htm>. Accessed on 15 February, 2019.

Weber CR and Fehr WR (1966) Seed yield losses from lodging and combine harvesting in soybeans. Agronomy Journal 58: 287-289.

Wen ZH, Zeng YX, Ji ZJ and Yang CD (2015) Mapping quantitative trait loci for sheath blight disease resistance in Yangdao 4 rice. Genetics and Molecular Research 14: 1636-1649.

Ye WJ, Hu SK, Wu LW, Ge CW, Cui YT, Chen P, Xu J, Dong GJ, Guo LB and Qian $\mathrm{Q}$ (2017) Fine mapping a major QTL $q F C C 7$, for chlorophyll content in rice (Oryza sativa L.) cv. PA64s. Plant Growth Regulation 81:81-90.

Zeng YX, Wen ZH, Ma LY, Ji ZJ, Li XM and Yang CD (2013) Development of 1047 insertion-deletion markers for rice genetic studies and breeding. Genetics and Molecular Research 12:5226-5235.

Zeng Y, Ji Z, Wen Z, Liang Y and Yang C (2016) Combination of eight alleles at four quantitative trait loci determines grain length in rice. PLoS ONE 11: e0150832.

Zhou L, Liu S, Wu W, Chen D, Zhan X, Zhu A, Zhang Y, Sheng S, Cao L, Lou $X$ and $X u H$ (2016) Dissection of genetic architecture of rice plant height and heading date by multiple-strategy-based association studies. Scientific Reports 6: 29718. 